\title{
Kutscher, Nadia
}

\section{Digitale Ungleichheit als Herausforderung für Medienbildung}

Die deutsche Schule 111 (2019) 4, S. 379-390

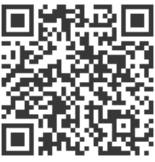

Quellenangabe/ Reference:

Kutscher, Nadia: Digitale Ungleichheit als Herausforderung für Medienbildung - In: Die deutsche

Schule 111 (2019) 4, S. 379-390 - URN: urn:nbn:de:0111-pedocs-206070 - DOI: 10.25656/01:20607

https://nbn-resolving.org/urn:nbn:de:0111-pedocs-206070

https://doi.org/10.25656/01:20607

in Kooperation mit / in cooperation with:

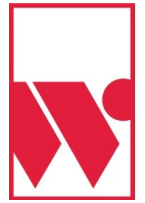

\section{WAXMANN}

www.waxmann.com

http://www.waxmann.com

\section{Nutzungsbedingungen}

Gewährt wird ein nicht exklusives, nicht übertragbares, persönliches und beschränktes Recht auf Nutzung dieses Dokuments. Dieses Dokument ist ausschließlich für den persönlichen, nicht-kommerziellen Gebrauch bestimmt. Die Nutzung stellt keine Übertragung des Eigentumsrechts an diesem Dokument dar und gilt vorbehaltlich der folgenden Einschränkungen: Auf sämtlichen Kopien dieses Dokuments müssen alle Urheberrechtshinweise und sonstigen Hinweise auf gesetzlichen Schutz beibehalten werden. Sie dürfen dieses Dokument nicht in irgendeiner Weise abändern, noch dürfen Sie dieses Dokument für öffentliche oder kommerzielle Zwecke vervielfältigen, öffentlich ausstellen, aufführen, vertreiben oder anderweitig nutzen.

Mit der Verwendung dieses Dokuments erkennen Sie die Nutzungsbedingungen an.

\section{Terms of use}

We grant a non-exclusive, non-transferable, individual and limited right to using this document.

This document is solely intended for your personal, non-commercial use. Use of this document does not include any transfer of property rights and it is conditional to the following limitations: All of the copies of this documents must retain all copyright information and other information regarding legal protection. You are not allowed to alter this document in any way, to copy it for public or commercial purposes, to exhibit the document in public, to perform, distribute or otherwise use the document in public.

By using this particular document, you accept the above-stated conditions of use.

\section{Kontakt / Contact:}

\section{peDOCS}

DIPF | Leibniz-Institut für Bildungsforschung und Bildungsinformation Informationszentrum (IZ) Bildung

E-Mail: pedocs@dipf.de

Internet: www.pedocs.de

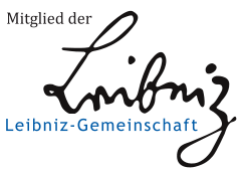




\section{DIGITALISIERUNG UND \\ BILDUNGSGERECHTIGKEIT}

DDS - Die Deutsche Schule

111. Jahrgang 2019, Heft 4, S. 379-390

https://doi.org/10.31244/dds.2019.04.02

(C) 2019 Waxmann

Nadia Kutscher

\section{Digitale Ungleichheit als Herausforderung für Medienbildung}

\section{Zusammenfassung}

Digitale Medien werden als relevant für gesellschaftliche Teilhabe in formalen, nonformalen und informellen Bildungskontexten betrachtet. Dieser Beitrag fokussiert unterschiedliche Dimensionen der (Re-)Produktion digitaler Ungleichheit in und zwischen Familie, Kinder- und Jugendhilfe sowie Schule und schlägt eine analytisch-reflexive Perspektive von Medienbildung vor, die Subjekt wie Strukturen in den Blick nimmt.

Schlüsselwörter: digitale Ungleichheit, digitale Medien, Medienbildung

\section{Digital Inequality as a Challenge for Media Education}

\section{Abstract}

Digital media is considered relevant for social participation in formal, non-formal and informal educational contexts. This paper focuses on different dimensions of the (re-) production of digital inequality in and between family, child and youth welfare as well as school and proposes an analytical-reflexive perspective of media education that looks at both subject and structures.

Keywords: digital inequality, digital media, media education

\section{Digitalisierung des Alltags und die Frage nach Teilhabechancen}

Digitale Medien sind seit Jahren Teil des Alltags von Kindern, Jugendlichen und Familien (vgl. Selwyn, 2003; Henrichwark, 2009; Livingstone, Haddon, Görzig \& Ólafsson, 2011; Aufenanger, 2013; Wagner, Gebel \& Lampert, 2013; Feil \& Grobbin, 2014; DIVSI, 2014, 2015, 2018; Kutscher \& Farrenberg, 2017; MPFS, 2018). Für Kinder stellt die Familie dabei den ersten Mediensozialisationsort dar; später kommen institutionelle Kontexte wie Kindertageseinrichtungen, Schule, Kinder- und 
Jugendhilfe sowie Peerbezüge hinzu, die im Jugendalter entsprechend an Relevanz gewinnen. Während im Zusammenhang mit der zunehmenden Durchdringung des Alltags mit digitalen Medien von einer „Online-offline-Hybridität“ (Hugger, 2009, S. 286) gesprochen werden kann, die impliziert, dass eine bewusste Trennung zwischen dem Handeln „innerhalb“ der digitalen Medien und „außerhalb“ für die meisten Menschen kaum mehr relevant oder möglich erscheint (vgl. Wagner \& Eggert, 2013, S. 15), wird vor dem Hintergrund empirischer Befunde deutlich, dass die Frage nach Teilhabe und Autonomieförderung durch digitale Medien erst auf der Basis ungleichheitsbezogener Analysen beantwortbar ist. Studien zur Mediennutzung junger Menschen betonen die autonomiebezogenen Potenziale digitaler Medien. So machen sie in diesen Zusammenhängen neue und spezifische Autonomieerfahrungen, indem sie sich in der Gestaltung sozialer Beziehungen austesten, unterschiedliche Formen von Selbstbestimmung und Beteiligung realisieren, Anerkennung im Peerkontext erfahren und (je nach Perspektive und Handlungszusammenhang begrenzte) Handlungswirksamkeit erleben und Mobilität praktizieren (vgl. Lauber, Wagner \& Theunert, 2007, S. 34 ff.; Brüggen, 2011). Im Kontext dieser frühen und teils weitgehenden Autonomieerfahrungen im Rahmen der digitalen Mediennutzung entstehen jedoch auch neue Herausforderungen sowohl für Kinder und Jugendliche als auch für Eltern und pädagogische Fachkräfte, die junge Menschen im Aufwachsen begleiten (vgl. Deutscher Bundestag, 2013, S. 179). Diese sind einerseits mit Risiken digitaler Räume und sich darin entfaltender Handlungsoptionen verbunden (vgl. Livingstone et al., 2011) und betreffen andererseits das Spannungsfeld von Autonomieermöglichung und Schutzaufgaben im Rahmen von Erziehung und Bildung, die nicht nur das Handeln der jungen Menschen, sondern auch der für sie Verantwortlichen betreffen (vgl. Kutscher \& Bouillon, 2018, S. 13).

Parallel zu dieser Entwicklung findet sich seit Jahren in der Wissenschaft und der Öffentlichkeit ein Diskurs darüber, inwiefern damit Chancen oder Risiken verbunden sind, welche Teilhabeoptionen sich durch digitale Mediennutzung in der Gesellschaft eröffnen und welche Bedeutung dem Aufwachsen mit digitalen Medien in diesem Zusammenhang zuzumessen ist (vgl. Spitzer, 2006, 2012; Schmidt, PausHasebrink \& Hasebrink, 2011; Wagner, 2013). Digitale Medien werden mittlerweile in vielen Alltagskontexten nicht nur vielfach genutzt, sondern ihre (kompetente) Nutzung wird auch zunehmend in bildungsinstitutionellen Zusammenhängen wie auch in der Arbeitswelt vorausgesetzt und zur Teilhabefrage in gesellschaftlichen Zusammenhängen. Idealistische Vorstellungen vom Internet (vgl. Tapscott, 1998; Palfrey \& Gasser, 2008) gingen lange Zeit von der These aus, dass das Internet freien und gleichen Zugang für alle Menschen zu Informationen, Wissen und gesellschaftlicher Teilhabe eröffne und darüber hinaus per se zu einer Inkorporierung demokratischer Strukturen führe (vgl. Palfrey \& Gasser, 2008, S. 309 ff.). Diese utopischen Ideen einer digitalisierten Zukunftsgesellschaft werfen vor dem Hintergrund empirischer Befunde zur ungleichen Verteilung gesellschaftlicher Teilhabeoptionen im Zusammenhang mit digitaler Mediennutzung die Frage auf, unter welchen 
Umständen diese Teilhabe realisiert werden kann. Sofern gesellschaftliche Teilhabe mit Bezug auf Kategorien der Sozial- und Armutsberichtserstattung als die Eröffnung von Möglichkeiten der Teilnahme an Kultur und Bildung - verbunden mit einem entsprechenden Rechtsstatus, der u.a. politische Partizipation und Teilhabe an sozialen Dienstleistungen umfasst, der Einbettung in soziale Nahbeziehungen und der Möglichkeit zur Erwerbsbeteiligung - verstanden wird (vgl. Bartelheimer, 2007, S. 10), ist also zu fragen, inwiefern und unter welchen Umständen die Nutzung digitaler Medien dies ermöglicht und welche Widersprüche sich auch im Kontext des Postulats von Medienbildung zeigen. Die Frage der Teilhabeermöglichung durch Informationszugang, Kommunikation, Beteiligung an politischen wie privaten Aktivitäten und Meinungsbildungsprozessen und Bildungszugänge wird im Folgenden mit Fokus auf spezifische Phänomene und Entwicklungen im Zusammenhang mit digitalen Ungleichheitsphänomenen diskutiert.

\section{Digitale Ungleichheit - Dimensionen}

Die Mediennutzung in Kindheit und Jugend ist durch den familialen Alltag, elterliche Ressourcen und Peerbeziehungen geprägt. Medienerziehungsstile und Medienpraxen werden dabei unterschiedlich ausgestaltet, sodass sowohl familial vorhandenes als auch über soziale Netzwerkbeziehungen verfügbares kulturelles Kapital, ungleiche Nutzungsweisen digitaler Medien und mediale Habitus und damit auch ihre bildungsrelevante Anschlussfähigkeit entsprechend $\mathrm{zu}$ einer Reproduktion ungleicher Bildungschancen beitragen (vgl. Lareau, 2011; Angus, Snyder \& Sutherland-Smith, 2004; Angus, 2007; Feil, Gieger \& Grobbin, 2010; Schmidt et al., 2011; Wagner et al., 2013; Paus-Hasebrink, Kulterer \& Sinner, 2019). Dieses als digitale Ungleichheit gekennzeichnete Phänomen bezeichnet - als weitere Differenzierung nach der Debatte um die „digitale Kluft“ und über die „Wissenskluft-Hypothese“ (Tichenor, Donohue \& Olien, 1970) hinausgehend - Ungleichheiten innerhalb der Mediennutzung (vgl. DiMaggio \& Hargittai, 2001), die auf der ungleichen Verfügbarkeit von ökonomischem, kulturellem und sozialem Kapital (Bourdieu, 1983) als prägenden Faktoren für Präferenzen, habituelle Fähigkeiten und strukturelle Möglichkeiten in der Mediennutzung beruhen. Dabei entfaltet sich die faktische Nutzung abhängig von den Aufmerksamkeits- und Navigationsentscheidungen vor dem Hintergrund von (sozial kontextualisierten) Interessen, Wissen, Fähigkeiten und Möglichkeiten der Nutzer*innen, so dass innerhalb der neuen Medien eine kulturelle und soziale Differenzierung habitualisierter Nutzungspraktiken entsteht, die auch Beteiligungsungleichheiten bedingt (vgl. Rudolph, 2019, S. 306 f; DIVSI, 2014, S. 99 ff.; Deutscher Bundestag, 2013, S. 183; Forschungsverbund DJI \& TU Dortmund, 2011; Klein, 2008, S. $511 \mathrm{ff}$.).

1 Hierbei war vor allem die sozial ungleiche Verfügbarkeit von Hard- und Software als technische Voraussetzungen des Zugangs zum Internet gemeint. 
Das bedeutet, die materiellen, kulturellen und sozialen Ressourcen, die Nutzer*innen außerhalb digitaler Medien zur Verfügung stehen, sind dabei ebenso relevant wie die Nutzungspraktiken und -erfahrungen selbst (vgl. Kutscher, 2018, S. 1436). Im Zusammenhang mit diesen sozial ungleichen Medienpraktiken sind darüber hinaus „Inhalte und spezifische Interaktionsformen und Machtrelationen, Ein- und Ausschließungsprozesse sowie Partizipationsprozesse“ (Tillmann, 2008, S. 94 f.) relevant, durch die auf kommunikativem Wege soziale Ungleichheit und Exklusion, auch zwischen Nutzer*innen, reproduziert werden. Dabei sind Differenzkategorien wie Geschlecht, Ethnizität, Alter, regionale Lage, Bildung und Beeinträchtigungen auf unterschiedliche Weise relevant; von zentraler Bedeutung sind Bildungsungleichheiten (vgl. Rudolph, 2019, S. 162 ff.). Diese „nutzer*innenseitigen“ ungleichen „kategorialen" Bedingungen und zeitlichen, materiellen, mentalen, sozialen und kulturellen Ressourcen (vgl. van Dijk, 2005, S. 9 ff.) verknüpfen sich mit strukturellen Bedingungen wie Zugang zu digitalen Technologien und führen $\mathrm{zu}$ ungleichen Teilhabeoptionen (vgl. ebd., S. 163 ff.).

$\mathrm{Zu}$ diesen zwischen digitalem Angebot und ressourcenbedingten ungleichen Nutzungsweisen und -fähigkeiten sich entfaltenden Aspekten digitaler Ungleichheit kommt im Zuge der Etablierung algorithmenbasierter Metadatenauswertung und infrastruktureller Zugangsdifferenzierung eine weitere Dimension hinzu: die als Zero- oder Third-Level-Divide (vgl. Zorn, 2017; Verständig, Klein \& Iske, 2016) bezeichnete infrastrukturelle Ungleichheit (vgl. Iske \& Kutscher, 2020). Es geht dabei - neben einer nicht garantierten Netzneutralität, die eine ungleiche Priorisierung bestimmter Dateninhalte von Telekommunikationsanbietern über das Internet und damit die Stratifizierung von Zugang zu Inhalten im Netz mit sich bringen kann - um die datenbasierte Kategorisierung von Nutzer*innen, die für deren Teilhabe in ökonomischer wie gesellschaftlicher Hinsicht teils heute schon weitreichende Folgen hat. Die Reproduktion von sozialer Diskriminierung im Zuge algorithmischer Berechnungen führt dabei zu kumulativen Benachteiligungen (vgl. Gandy, 2011, S. 176), sodass damit Nutzer*innen zu „big data rich“ und „big data poor“ werden, die aufgrund des Werts ihrer Daten und deren Gewichtung im Zuge algorithmenbasierter Zugangssteuerungs- und Ratingprozesse unterschiedliche ökonomische und gesellschaftliche Teilhabeoptionen erhalten (vgl. Christl, 2017).

In die Geräte eingeschrieben finden sich ebenfalls ungleichheitsrelevante Strukturen. Dinuka Wijetunga bezeichnet dies als „Objektifizierung“ (Wijetunga, 2014, S. 712). Damit ist gemeint, dass im Rahmen der Entwicklung von Hard- und Software die technische Konstruktion von Apps oder digitalen Geräten auf spezifischen Annahmen über die künftigen Nutzer*innen beruht. Empirische Studien zeigen, dass sich die Nutzungsbandbreite u. a. von Smartphones und den darauf befindlichen Optionen und Apps erst auf der Basis von Wissens- und Fähigkeitsvoraussetzungen entfaltet, die abhängig von kulturellem Kapital ungleich verfügbar sind, sodass damit auch das Potenzial besteht, dass sich Ungleichheiten verstärken. Dies zeigt sich beispielsweise 
auch in Studien zur Mediennutzung Geflüchteter, die als unbegleitete Minderjährige Adressat*innen der Kinder- und Jugendhilfe sind (vgl. Fendrich \& Tabel, 2018, S. 20). Für sie ist der Zugang zu Internet und digitalen Medien einerseits grundlegend für die Aufrechterhaltung des Kontakts zur Herkunftsfamilie sowie für die Orientierung in der Aufnahmegesellschaft (vgl. Kutscher \& Kreß, 2018, 2019; Emmer, Richter \& Kunst, 2016; Gillespie et al., 2016). Andererseits sind medienbezogene Fähigkeiten auch bei den jungen Geflüchteten durchaus unterschiedlich vorhanden und u. a. auch vom kulturellen, sozialen und ökonomischen Kapital ihrer Herkunftsfamilie abhängig (vgl. Kutscher \& Kreß, 2018, S. 7).

\section{Medienbildung und öffentliche Institutionen}

Die internationale Vergleichsstudie „International Computer and Information Literacy Study“ (ICILS) zeigt, dass schulische Kompetenzen im Zusammenhang mit digitaler Mediennutzung stark von der sozialen Herkunft beeinflusst sind und zur Benachteiligung deprivilegierter Schüler*innen führen (vgl. Bos et al., 2014, S. 25). Darüber hinaus führen im Zusammenspiel von Familie und Schule Inkompatibilitäten medialer Habitus zur Reproduktion der Benachteiligung sozial deprivilegierter Kinder in der Grundschule (vgl. Henrichwark, 2009), und eine Reihe von Studien verweisen darauf, dass digitale Medienbildung auch in der Schule noch ein weitgehendes Desiderat darstellt (vgl. Breiter, Welling \& Stolpmann, 2010; Breiter, Aufenanger, Averbeck, Welling \& Wedjelek, 2013; Brüggemann, 2013; Fraillon, Ainley, Schulz, Friedman \& Gebhardt, 2014; Bertelsmann Stiftung, 2017a, 2017b). Auch im Kita-Bereich werden Medienpraktiken sozial benachteiligter Familien von Seiten der Fachkräfte eher skeptisch betrachtet (vgl. Bischof \& Kutscher, 2019). Im Feld der Kinder- und Jugendhilfe zeigt sich ebenfalls ein teils disparates Bild. Während dort einerseits digitale Medien in Alltagskommunikation längst eine Rolle spielen (mit allen Widersprüchen und Fragen, die sich rund um Datenschutz- und andere Fachlichkeitsstandards stellen - vgl. Kutscher, Ley, Seelmeyer, Siller, Tillmann \& Zorn, 2019), stellen eine entsprechende Qualifizierung von Fachkräften sowie die Reflexion ungleichheitsbezogener Fragen Desiderata dar. In vielen Einrichtungen der Kinder- und Jugendhilfe findet sich - ähnlich wie auch in manchen Schulen kaum eine grundlegende Ausstattung mit digitalen Medien wie Computern oder Internetzugängen (vgl. Kutscher \& Kreß, 2015). Damit besteht neben einer digitalen Ungleichheit in Bezug auf die Nutzung (s.u.) auch eine institutionelle Zugangskluft, die dazu führt, dass basale Dinge wie das Bewältigen von Hausaufgaben in stationären Erziehungshilfeeinrichtungen nur unter eingeschränkten Bedingungen realisiert werden können.

Während (medien-)bildungspolitische Debatten eine verstärkte Zuständigkeit öffentlicher Bildungseinrichtungen thematisieren und Pilotprojekte auf Landesebene (beispielsweise in Bayern und NRW) auch im Kita-Bereich die Qualifizie- 
rung für Medienbildung befördern sollen, gehen diese Entwicklungen einher mit einem Diskurs über Aufwachsen in öffentlicher Verantwortung, in dem den Bildungseinrichtungen die Rolle als Ort der Kompensation familialer Benachteiligungen zugeschrieben wird. Empirische Studien zeigen jedoch, dass - obwohl digitale Medienerfahrungen von Kindern biographisch immer früher beginnen - beispielsweise Kindertageseinrichtungen weniger als Orte der Medienerziehung in den Blick geraten als die Schule (vgl. Bischof \& Kutscher, 2019; Wagner et al., 2013). Untersuchungen zur Medienerziehung in der Kita verweisen darauf, dass Orientierungslosigkeit in Kindertageseinrichtungen und Ausbildungsstätten, Unsicherheit der Erzieher*innen in Bezug auf den Medienumgang von Kindern und Familien und Medienerziehung in der Kindertageseinrichtung, mangelnde eigene Medienerfahrung, Sensibilisierung und medienerzieherisches Wissen das Feld prägen (vgl. Six \& Gimmler, 2007; Friedrichs-Liesenkötter, 2016). Studien zu digitalen Medien und Schule weisen vor allem auf infrastrukturelle und qualifikationsbezogene Probleme hin (vgl. Bertelsmann Stiftung, 2017a, 2017b; Bos et al., 2014). Somit sind mit den beruflichen Habitus von Fachkräften (vgl. Friedrichs-Liesenkötter, 2016), institutionellen Bedingungen (vgl. Bos et al., 2014, S. 17 ff.), erzieherischen Vorstellungen von Schutzräumen in der frühen Kindheit (vgl. Schubert, Brüggen, Oberlinner, Eggert \& Jochim, 2018, S. 28), Bildern von Jugend (vgl. Deutscher Bundestag, 2017, S. 462) und Diskursen über öffentliche und private Erziehungsverantwortung (vgl. Kutscher \& Schmidt, 2019) sowohl subjektive als auch gesellschaftliche Dimensionen der Verhandlung von Erziehungsvorstellungen, Kindheits- bzw. Jugendkonstrukten und Verantwortungsdistribution relevant dafür, auf welche Weise digitale Medienerziehung und -bildung in öffentlichen Bildungsinstitutionen verhandelt werden.

\section{Subjektbezogene Medienbildung, linking social capital und politische Steuerung}

Wie gezeigt wurde, hat kulturelles und soziales Kapital eine bedeutsame Rolle im Kontext der digitalen Ungleichheitsreproduktion, verknüpft mit strukturellen Bedingungen, die auf gesellschaftlicher Ebene zu impliziten Steuerungsmechanismen ungleicher Teilhabe werden. Häufig wird in bildungspolitischen und -praktischen Debatten ein „Mehr an Medienkompetenzförderung“ gefordert, doch es wird kaum reflektiert, inwiefern solch eine subjektbezogene, auf Kompetenzen fokussierte Intervention strukturelle Hindernisse wie metadatenbezogene Ungleichbehandlung, gesellschaftliche Ungleichheiten oder auch infrastrukturelle Disparitäten überwinden kann.

Alexandra Klein bietet mit ihrem Modell der medialen, interpersonalen und inhaltlichen Passung als Voraussetzung für eine faktisch realisierte Nutzung digitaler Angebote einen analytischen Fokus, der die Nutzer*innenpräferenzen in Relation zu digitalen Angebotsstrukturen setzt (vgl. Klein, 2008, S. 533). Erst wenn beides zuein- 
ander „passt“, wird ein Angebot auch tatsächlich nutzbar. Unter der Berücksichtigung lebensweltlich unterschiedlicher Präferenzen und Fähigkeiten (vgl. Zillien, 2008; Kompetenzzentrum Informelle Bildung, 2007) wird dann analytisch rekonstruierbar, weshalb sich die Nutzung innerhalb digitaler Angebote wie beispielsweise auch sozialer Netzwerke ausdifferenziert. Habituell unterschiedliche Präferenzen und Fähigkeiten können somit nicht einfach durch eine Vermittlung weiteren medienbezogenen Wissens oder bestimmter Kompetenzen verändert werden, sondern bedürfen längerer Prozesse in einem Feld, das auch über soziale Beziehungen andere Fähigkeiten eröffnet. In diesem Zusammenhang rücken Bildungsinstitutionen in den Blick, als Räume, innerhalb derer über „weak ties“ (Granovetter, 1983) und „linking social capital“ (Szreter \& Woolcock, 2004) - also schwache Bindungen, über die erweiterte Ressourcen zugänglich werden - über eine kontinuierliche Flankierung durch Fachkräfte entsprechende Prozesse begleitet werden können. Allerdings ist die Frage, inwiefern dabei Aspekte der „kulturellen Legitimität“ (Schäffer, 2000) verhandelt und reflektiert werden, wenn viele Angebote im pädagogischen Bereich voraussetzungsvoll, an einem ressourcenreichen zweckrational orientierten Habitus orientiert ausgestaltet sind (vgl. Welling, 2008). Verschiedene Medienkompetenzmodelle gehen von spezifischen "legitimen“ Nutzungsweisen aus, die anzueignen, und anderen, die zu überwinden sind (vgl. Schäfer \& Lojewski, 2007, S. 9, 92). Dabei werden habituelle Differenzen ausgeblendet und bestimmte Praktiken als legitime auf- und andere abgewertet, indem Differenzierungen von „aktiver, produktiver“ oder richtiger/falscher Mediennutzung entkontextualisiert und an einem ressourcenreichen Habitus ausgerichtet gesetzt werden. Damit wird das Feld im Sinne Bourdieus, d.h. der lebensweltliche Alltag, als Bedingung für die Aneignung bestimmter Fähigkeiten und Bezugsrahmen für die Sinnhaftigkeit von Praktiken nicht hinreichend reflektiert, so dass dies zu einer impliziten Ungleichheitsreproduktion im Rahmen von Bildungsanstrengungen führen kann (vgl. Kutscher, 2009). Über eine alltagshermeneutische Rekonstruktion dieser ungleichen Voraussetzungen und deren Passung zum Angebot, die diese lebensweltlichen Bedingungen und deren Konsequenzen für die Gestaltung pädagogischer Arrangements ausdrücklich reflektiert, können mit einer entsprechenden personalen Begleitung, mit Hilfe materieller Ressourcen und in Form einer kompensatorischen Beziehungsarbeit die Teilhabe-Verwirklichungschancen im Sinne interner und externer Capabilities (vgl. Robeyns, 2006), d. h. sowohl subjektiver Fähigkeiten als auch struktureller Bedingungen, erweitert werden.

Die dargestellten Dimensionierungen digitaler Ungleichheit verweisen auf die Grenzen einer Bildung des Subjekts als systematischem Ort der Überwindung von Ungleichheit. Vielmehr wird deutlich, dass neben einer subjektbezogenen Intervention eine strukturell-politische Steuerung der Kontexte und Strukturen erforderlich ist. 


\section{Literatur und Internetquellen}

Angus, L. (2007). Implications for Social Inequality in Internet Use for Educational Policies and Programs. In Kompetenzzentrum Informelle Bildung (Hrsg.), Grenzenlose Cyberwelt? Zum Verhältnis von digitaler Ungleichheit und neuen Bildungszugängen für Jugendliche (S. 15-29). Wiesbaden: VS. https://doi.org/10.1007/978-3-531-90519-8_1

Angus, L., Snyder, I., \& Sutherland-Smith, W. (2004). ICT and Educational (Dis)Advantage: Cultural Resources and the Digital Divide. In G. Troman, R. Jeffrey \& G. Walford (Hrsg.), Ethnographies of Educational and Cultural Conflicts: Strategies and Resources (S. 45-66). London: Emerald. https://doi.org/10.1016/S1529-210X(04)09004-7

Aufenanger, S. (2013). Digitale Medien im Leben von Kindern zwischen null und fünf Jahren. medien + erziehung. zeitschrift für medienpädagogik, 57 (2), 8-14.

Bartelheimer, P. (2007). Politik der Teilhabe. Ein soziologischer Beipackzettel (FriedrichEbert-Stiftung (Hrsg.), Fachforum. Analysen und Kommentare). Zugriff am 13.09.2019. Verfügbar unter: https://library.fes.de/pdf-files/do/04655.pdf.

Bertelsmann Stiftung (Hrsg.). (2017a). Monitor Digitale Bildung. Digitales Lernen an Schulen. Zugriff am 13.09.2019. Verfügbar unter: https://www.bertelsmann-stiftung.de/ fileadmin/files/BSt/Publikationen/GrauePublikationen/BSt_MDB3_Schulen_web.pdf.

Bertelsmann Stiftung (Hrsg.). (2017b). Monitor Digitale Bildung. Digitales Lernen an Grundschulen. Verfügbar unter: https://www.bertelsmann-stiftung.de/fileadmin/files/ BSt/Publikationen/GrauePublikationen/BST_DigiMonitor_Grundschulen.pdf.

Bischof, J., \& Kutscher, N. (2019, im Erscheinen). Ergebnisse der wissenschaftlichen Begleitung des Projekts „Medienbildung in der Kita“. Abschlussbericht.

Bos, W., Eickelmann, B., Gerick, J., Goldhammer, F., Schaumburg, H., Schwippert, K., Senkbeil, M., Schulz-Zander, R., \& Wendt, H. (Hrsg.). (2014). ICILS 2013. Computerund informationsbezogene Kompetenzen von Schülerinnen und Schülern in der 8. Jahrgangsstufe im internationalen Vergleich. Münster: Waxmann.

Bourdieu, P. (1983). Ökonomisches Kapital, kulturelles Kapital, soziales Kapital. In R. Kreckel (Hrsg.), Soziale Ungleichheiten (S. 183-198). Göttingen: Schwartz.

Breiter, A., Aufenanger, S., Averbeck, I., Welling, S., \& Wedjelek, M. (2013). Medienintegration in Grundschulen. Untersuchung zur Förderung von Medienkompetenz und der unterrichtlichen Mediennutzung in Grundschulen sowie ihrer Rahmenbedingungen in Nordrhein-Westfalen. Berlin: VISTAS.

Breiter, A., Welling, S., \& Stolpmann, B. E. (2010). Medienkompetenz in der Schule. Integration von Medien in den weiterführenden Schulen in Nordrhein-Westfalen. Berlin: VISTAS. Zugriff am 13.09.2019. Verfügbar unter: http://lfmpublikationen.lfm-nrw.de/ index.php?view=search\&search=Medienkompetenz+in + der+Schule.

Brüggemann, M. (2013). Digitale Medien im Schulalltag. Eine qualitativ rekonstruktive Studie zum Medienhandeln und berufsbezogenen Orientierungen von Lehrkräften. München: kopaed.

Brüggen, N. (2011). Facebook \& Co. Digitalisierte Lebenswelten - reale Problemwelten. Vortrag auf dem Onlineberatungsforum 2011 in Nürnberg. Verfügbar unter: www. ohm-hochschule.de/fileadmin/Fachbereiche/sw/Studienwerkstatt/Onlineberatung/Fach forum_2011/Brueggen_Facebook_co.pdf.

Christl, W. (2017). Corporate Surveillance in Everyday Life. How Companies Collect, Combine, Analyze, Trade, and Use Personal Data on Billions. A Report by Cracked Labs, Vienna, June 2017. Zugriff am 13.09.2019. Verfügbar unter: https://crackedlabs. org/dl/CrackedLabs_Christl_CorporateSurveillance.pdf.

Deutscher Bundestag (2013). 14. Kinder- und Jugendbericht der Bundesregierung. Bundestagsdrucksache 17/12200 vom 30.01.2013. Zugriff am 13.09.2019. Verfügbar unter: https://www.bmfsfj.de/blob/jump/93146/14-kinder-und-jugendbericht-data.pdf. 
Digitale Ungleichheit als Herausforderung für Medienbildung |

Deutscher Bundestag (2017). 15. Kinder- und Jugendbericht der Bundesregierung. Bundestagsdrucksache 18/11050 vom 01.02.2017. Zugriff am 13.09.2019. Verfügbar unter: https://www.bmfsff.de/blob/115438/d7ed644e1b7fac4f9266191459903c62/15-kinderund-jugendbericht-bundestagsdrucksache-data.pdf.

DiMaggio, P., \& Hargittai, E. (2001). From the "Digital Divide" to "Digital Inequality": Studying Internet Use as Penetration Increases. Princeton University Center for Arts and Cultural Policy Studies, Working Paper Series, No. 15. Verfügbar unter: https:// www.princeton.edu/ artspol/workpap/WP15\%20-\%20DiMaggio\%2BHargittai.pdf.

DIVSI $(2014,2015,2018)$. Studien des Deutschen Instituts für Vertrauen und Sicherheit im Internet (DIVSI). Zugriff am 13.09.2019. Verfügbar unter: www.divsi.de.

Emmer, M., Richter, C., \& Kunst, M. (2016). Flucht 2.0. Mediennutzung durch Flüchtlinge vor, während und nach der Flucht. Zugriff am 13.09.2019. Verfügbar unter: http://www. polsoz.fu-berlin.de/kommwiss/arbeitstellen/internationale_kommunikation/Media/ Flucht-2_0.pdf.

Feil, C., Gieger, C., \& Grobbin, A. (2010). Kernaussagen und zentrale Ergebnisse des DJIProjekts „Digital Divide“ (Auf einen Blick). DJI Online Thema 2010/02: Digital kompetent oder abgehängt? Wege von Kindern und Jugendlichen ins Netz. Verfügbar unter: http://www.dji.de/themen/dji-top-themen/dji-online-februar-2010-digital-kompetentoder-abgehaengt-wege-von-kindern-und-jugendlichen-ins-netz/auf-einen-blick.html.

Feil, C., \& Grobbin, A. (2014). Digitale Medien: Beratungs-, Handlungs- und Regulierungsbedarf aus Elternperspektive. Kurzbericht zur Teilstudie - Eltern mit 1- bis 8-jährigen Kindern. München: Deutsches Jugendinstitut.

Fendrich, S., \& Tabel, A. (2018). Rückgang bei neu begonnenen Fremdunterbringungen. Komdat, (3), 16-19.

Forschungsverbund DJI \& TU Dortmund (2011). Jugendliche Aktivitäten im Wandel. Gesellschaftliche Beteiligung und Engagement in Zeiten des Web 2.0. Endbericht Forschungsverbund Deutsches Jugendinstitut \& Technische Universität Dortmund. Verfügbar unter: http://www.forschungsverbund.tu-dortmund.de/fileadmin/Files/En gagement/Abschlussbericht_Engagement_2_0.pdf.

Fraillon, J., Ainley, J., Schulz, W., Friedman, T., \& Gebhardt, E. (2014). Preparing for Life in a Digital Age. The IEA International Computer and Information Literacy Study International Report. Zugriff am 13.09.2019. Verfügbar unter: http://www.iea.nl/icils_2013. html.

Friedrichs-Liesenkötter, H. (2016). Medienerziehung in Kindertagesstätten: Habitusformationen angehender ErzieherInnen. Wiesbaden: Springer VS. https://doi.org/10.1007/9783-658-12307-9

Gandy, O. (2011). Consumer Protection in Cyberspace. tripleC. Journal for a Global Sustainable Information Society, 9 (2), 175-189. https://doi.org/10.31269/triplec.v9i2.267

Gillespie, M., Ampofo, L., Cheesman, M., Faith, B., Iliadou, E., Issa, A., Osseiran, S., \& Skleparis, D. (2016). Mapping Refugee Media Journey. Smartphones and Social Media Networks. Zugriff am 13.09.2019. Verfügbar unter: http://www.open.ac.uk/ccig/sites/ www.open.ac.uk.ccig/files/Mapping\%20Refugee\%20Media\%20Journeys\%2016\%20 May\%20FIN\%20MG_0.pdf.

Granovetter, M. (1983). The Strength of Weak Ties: A Network Theory Revisited. Sociological Theory, 1, 201-233. https://doi.org/10.2307/202051

Henrichwark, C. (2009). Der bildungsbezogene mediale Habitus von Grundschulkindern. Eine empirische Studie zur Reproduktion sozialer Ungleichheit in Schule und Familie. Dissertation Universität Wuppertal. Zugriff am 13.09.2019. Verfügbar unter: http:// elpub.bib.uni-wuppertal.de/servlets/DocumentServlet?id=1025.

Hugger, K.-U. (2009). Junge Migranten online: Suche nach sozialer Anerkennung und Vergewisserung von Zugehörigkeit. Wiesbaden: Springer VS. 
Iske, S., \& Kutscher, N. (2020, im Erscheinen). Digitale Ungleichheiten im Kontext Sozialer Arbeit. In N. Kutscher, T. Ley, U. Seelmeyer, F. Siller, A. Tillmann \& I. Zorn (Hrsg.), Handbuch Digitalisierung und Soziale Arbeit. Weinheim: Beltz Juventa.

Klein, A. (2008). Soziales Kapital Online. Soziale Unterstützung im Internet. Eine Rekonstruktion virtualisierter Formen sozialer Ungleichheit. Dissertation Universität Bielefeld. Verfügbar unter: http://bieson.ub.uni-bielefeld.de/volltexte/2008/1260/.

Kompetenzzentrum Informelle Bildung (2007). Grenzenlose Cyberwelt? Zum Verhältnis von digitaler Ungleichheit und neuen Bildungszugängen für Jugendliche. Wiesbaden: Springer VS .

Kutscher, N. (2009). Ungleiche Teilhabe - Überlegungen zur Normativität des Medienkompetenzbegriffs. Zeitschrift MedienPädagogik, 17, 1-18. https://doi.org/10.21240/ mpaed/17/2009.04.17.X.

Kutscher, N. (2018). Soziale Arbeit und Digitalisierung. In H.-U. Otto, H. Thiersch, R. Treptow \& H. Ziegler (Hrsg.), Handbuch Soziale Arbeit (6., überarb. Aufl.) (S. 14301440). München: Reinhardt.

Kutscher, N., \& Bouillon, R. (2018). Kinder. Bilder. Rechte. Persönlichkeitsrechte von Kindern im Kontext der digitalen Mediennutzung in der Familie. Studie in Kooperation mit dem Deutschen Kinderhilfswerk e.V. Berlin: Deutsches Kinderhilfswerk e.V. Zugriff am 13.09.2019. Verfügbar unter: https://www.dkhw.de/fileadmin/Redaktion/1_Unsere_ Arbeit/1_Schwerpunkte/6_Medienkompetenz/6.13._Studie_Kinder_Bilder_Rechte/ DKHW_Schriftenreihe_4_KinderBilderRechte.pdf.

Kutscher, N., \& Farrenberg, D. (2017). Teilhabe und soziale Kompetenz durch die Nutzung von digitalen Medien: Herausforderungen für die Kinder- und Jugendpolitik. Expertise für den 10. Kinder- und Jugendbericht des Landes Nordrhein-Westfalen. Zugriff am 13.09.2019. Verfügbar unter: https://www.mfkjks.nrw/sites/default/files/asset/docu ment/10-kjbnrw-expertise-kutscher_farrenberg_u.a.pdf.

Kutscher, N., \& Kreß, L.-M. (2015). Internet ist gleich mit Essen. Empirische Studie zur Nutzung digitaler Medien durch unbegleitete minderjährige Flüchtlinge. Projektbericht in Zusammenarbeit mit dem Deutschen Kinderhilfswerk. Vechta: Universität. https://doi. org/10.13140/RG.2.1.1028.8729

Kutscher, N., \& Kreß, L.-M. (2018). The Ambivalent Potentials of Social Media Use by Unaccompanied Minor Refugees. Social Media \& Society, 4 (1), Special Issue: Forced Migrants and Digital Connectivity, 1-10. https://doi.org/10.1177/2056305118764438

Kutscher, N., \& Kreß, L.-M. (2019). Das Smartphone als Schlüsselmedium - Transnationale und lokale Teilhabe unbegleiteter geflüchteter Jugendlicher. Migration und Soziale Arbeit, 40 (4), 325-330.

Kutscher, N., Ley, T., Seelmeyer, U., Siller, F., Tillmann, A., \& Zorn, I. (Hrsg.). (2019). Handbuch Digitalisierung und Soziale Arbeit. Weinheim: Beltz Juventa.

Kutscher, N., \& Schmidt, F. (2019). Verteilte Erziehungs- und Sorgeverantwortung im Kontext digitalisierungs- und ernährungsbezogener Wohlfahrtspraktiken. Zeitschrift für Soziologie der Erziehung und Sozialisation, 39 (2), 152-167.

Lareau, A. (2011). Unequal Childhoods: Class, Race, and Family Life (2., überarb. Aufl.). Berkeley, CA, Los Angeles, CA, \& London: University of California Press.

Lauber, A., Wagner, U., \& Theunert, H. (2007). Internetradio und Podcasts - neue Medien zwischen Radio und Internet. Eine explorative Studie zur Aneignung neuer Audioangebote im Auftrag der Bayerischen Landeszentrale für neue Medien (BLM). München: BLM. Verfügbar unter: www.jff.de/dateien/Endbericht_Internetradio_Podcasts1.pdf.

Livingstone, S., Haddon, L., Görzig, A., \& Ólafsson, K. (2011). Risks and Safety on the Internet: The Perspective of European Children: Full Findings and Policy Implications from the EU Kids Online Survey of 9-16 Year Olds and Their Parents in 25 Countries. 
London: EU Kids Online Network. Zugriff am 13.09.2019. Verfügbar unter: www.eu kidsonline.net; http://eprints.lse.ac.uk/33731/.

MPFS (Medienpädagogischer Forschungsverbund Südwest) (2018). Studie Jugend, Information, (Multi-)Media. Zugriff am 13.09.2019. Verfügbar unter: www.mpfs.de.

Palfrey, J., \& Gasser, U. (2008). Born Digital. New York: Basic Books.

Paus-Hasebrink, I., Kulterer, J., \& Sinner, P. (2019). Social Inequality, Childhood and the Media. London: Palgrave Macmillan. https://doi.org/10.1007/978-3-030-02653-0

Robeyns, I. (2006). The Capability Approach in Practice. Journal of Political Philosophy, 14 (3), 351-376. https://doi.org/10.1111/j.1467-9760.2006.00263.x

Rudolph, S. (2019). Digitale Medien, Partizipation und Ungleichheit. Eine Studie zum sozialen Gebrauch des Internets. Wiesbaden: Springer Nature. https://doi.org/10.1007/978-3658-26943-2

Schäfer, M., \& Lojewski, J. (2007). Internet und Bildungschancen. Die soziale Realität des virtuellen Raumes. München: kopaed.

Schäffer, B. (2000). Das Internet: ein Medium kultureller Legitimität in Bildungskontexten? In W. Marotzki, D. Meister \& U. Sander (Hrsg.), Zum Bildungswert des Internet (S. 2150). Opladen: Leske + Budrich. https://doi.org/10.1007/978-3-322-97472-3_13

Schmidt, J.-H., Paus-Hasebrink, I., \& Hasebrink, U. (Hrsg.). (2011). Heranwachsen mit dem Social Web. Berlin: VISTAS.

Schubert, G., Brüggen, N., Oberlinner, A., Eggert, S., \& Jochim, V. (2018). Haltungen von pädagogischem Personal zu mobilen Medien, Internet und digitalen Spielen in Kindertageseinrichtungen. Bericht der Teilstudie „Mobile Medien und Internet im Kindesalter - Fokus Kindertageseinrichtungen“. München: JFF - Institut für Medienpädagogik in Forschung und Praxis. Verfügbar unter: www.jff.de/mofam.

Selwyn, N. (2003). "Doing IT for the Kids": Re-Examining Children, Computers and the "Information Society". Media, Culture \& Society, 25 (3), 351-378. https://doi. org/10.1177/0163443703025003004

Six, U., \& Gimmler, R. (Hrsg.). (2007). Die Förderung von Medienkompetenz im Kindergarten: Eine empirische Studie zu Bedingungen und Handlungsformen der Medienerziehung. Berlin: VISTAS.

Spitzer, M. (2006). Vorsicht Bildschirm! Elektronische Medien, Gehirnentwicklung, Gesundheit und Gesellschaft. Stuttgart: Klett Lerntraining GmbH.

Spitzer, M. (2012). Digitale Demenz: Wie wir uns und unsere Kinder um den Verstand bringen. München: Droemer.

Szreter, S., \& Woolcock, M. (2004). Health by Association? Social Capital, Social Theory, and the Political Economy of Public Health. International Journal of Epidemiology, 33 (4), 650-667. https://doi.org/10.1093/ije/dyh013

Tapscott, D. (1998). Net Kids. Die digitale Generation erobert Wirtschaft und Gesellschaft. Wiesbaden: Gabler. https://doi.org/10.1007/978-3-322-82802-6

Tichenor, P. J., Donohue, G. A., \& Olien, C. (1970). Mass Media Flow and Differential Growth in Knowledge. Public Opinion Quarterly: Journal of the American Association for Public Opinion Research, 34 (2), 159-170. https://doi.org/10.1086/267786

Tillmann, A. (2008). Identitätsspielraum Internet. Weinheim \& München: Juventa.

van Dijk, J. (2005). The Deepening Divide. Inequality in the Information Society. Thousand Oaks, CA: Sage.

Verständig, D., Klein, A., \& Iske, S. (2016). Zero-Level Digital Divide: Neues Netz und neue Ungleichheiten. Si:So. Analysen - Berichte - Kontroversen (Univ. Siegen), 21 (1), 50-55.

Wagner, U. (Hrsg.). (2013). Familienleben: Entgrenzt und vernetzt?! (Schriftenreihe Interdisziplinäre Diskurse, Bd. 7). München: kopaed. 
Wagner, U., \& Eggert, S. (2013). Das Medienhandeln von Heranwachsenden. Konstanten und Veränderungen. Materialien zum 14. Kinder- und Jugendbericht. Verfügbar unter: http://www.dji.de/14_kjb/14-KJB-Expertise-Wagner-ua.pdf.

Wagner, U., Gebel, C., \& Lampert, C. (Hrsg.). (2013). Zwischen Anspruch und Alltagsbewältigung: Medienerziehung in der Familie (Schriftenreihe Medienforschung der Landesanstalt für Medien NRW (LfM), Bd. 72). Berlin: VISTAS.

Welling, S. (2008). Computerpraxis Jugendlicher und medienpädagogisches Handeln. München: kopaed.

Wijetunga, D. (2014). The Digital Divide Objectified in the Design: Use of the Mobile Telephone by Underprivileged Youth in Sri Lanka. Journal of Computer-Mediated Communication, 19 (3), 712-726. https://doi.org/10.1111/jcc4.12071

Zillien, N. (2008). Digitale Ungleichheit. Neue Technologien und alte Ungleichheiten in der Informations- und Wissensgesellschaft. Wiesbaden: VS. https://doi.org/10.1007/978-3531-91493-0

Zorn, I. (2017). Wie viel „App-Lenkung“ verträgt die digitalisierte Gesellschaft? Herausforderungen digitaler Datenerhebungen für die Medienbildung. In S. Eder, C. Mikat \& A. Tillmann (Hrsg.), Software takes command. Herausforderungen der "Datafizierung“ für die Medienpädagogik in Theorie und Praxis (S. 19-33). München: kopaed.

Nadia Kutscher, Prof. Dr., geb. 1972, Professorin für Erziehungshilfe und Soziale Arbeit an der Universität zu Köln.

Korrespondenzadresse: Lehrstuhl Erziehungshilfe und Soziale Arbeit, Department Heilpädagogik und Rehabilitation, Humanwissenschaftliche Fakultät, Universität zu Köln, Klosterstr. 79b, 50931 Köln

E-Mail: nadia.kutscher@uni-koeln.de 